\title{
Atom bond connectivity index of molecular graphs of alkenes and cycloalkenes
}

\begin{abstract}
The atom-bond connectivity ( $\mathrm{ABC}$ ) index is one of the recently most investigated degree based molecular structure descriptors that have applications in chemistry. For a graph $\mathrm{G}$, the

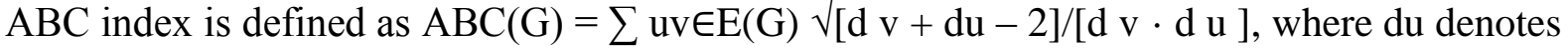
the degree of a vertex $u$ in $G$. In this paper, we establish the general formulas for the atom bond connectivity index of molecular graphs of alkenes and cycloalkenes.
\end{abstract}

Keyword: Alkenes and cycloalkenes; Atom bond connectivity index; Molecular graphs 